\title{
Sorgen und Hoffnungen der Allergologie in Deutschland und Europa
}

m letzten Heft des Jahres steht ein kurzer Rückblick an. 1998 war ein aktives Jahr: Zu den Höhepunkten zählten traditionelle Ereignisse wie die DGAI-Frühjahrstagung (J. Knop und G. Schultze-Werninghaus) in Mainz und der ÄDA-Kongreß (Th. Fuchs) in Göttingen. Aber auch Neues wurde geboten: So hat die Deutsche Akademie für Allergologie und Umweltmedizin (DAAU) erstmals offizielle zertifizierte Seminare durchgeführt (Themen: "Notfälle in der Allergologie“, „Hyposensibilisierung“) und ÄDA und DGAI initiierten gemeinsam unter der Federführung von J. Wenning eine Industrie-Plattform für Pressearbeit und Public Relations, die die Interessen von Allergie-Patienten und allergologisch tätigen Ärzten vertritt.

Sektionen und Arbeitsgruppen der DGAI haben ihre Arbeit aufgenommen bzw. weitergeführt. Besonders zu erwähnen sind die Arbeitsgruppen „Nahrungsmittel-Allergie“ (L. Jäger) und „Allergen-Karenz“ (H. Müsken).

Auch aus dem Forschungsbereich gibt es Positives zu berichten: Das von 1990 bis 1995 eingefrorene Forschungsschwerpunktprogramm „Allergie- und Lungenforschung" wurde endlich nach Fortschreibung in die Realität überführt. 1998 wurden in Deutschland fünf klinische Forschergruppen, davon drei für Allergie- und zwei für Lungenforschung eingerichtet: für die Allergologie in Freiburg (Sprecher E. Schöpf), Berlin (Sprecher: U. Wahn) und München (Sprecher: H. Behrendt, J. Ring und $\mathrm{H}$. Wagner), für die Lungenforschung in Bochum (Sprecher: G. Schultze-Werninghaus) und Marburg (Sprecher: E. von Wichert).

Nach Meinung der DGAI ist dies erfreulich, jedoch bei weitem nicht genug, um die Allergie-Forschung in Deutschland in der Breite wesentlich $\mathrm{zu}$ unterstützen. Es müssen an allen
Universitäten aktive allergologische Arbeitsgruppen entstehen.

In den meisten Bundesländern hat inzwischen die auf Betreiben der DGAI beschlossene Verlängerung der Weiterbildungszeit zum Erwerb der Zusatzbezeichnung „Allergologie“ (zwei Jahre) gegriffen. Dies wird für eine bessere Qualifizierung und Trennung von Spreu und Weizen unter den allergologisch tätigen Ärzten sorgen. Es wird nicht mehr gehen, daß an bestimmten Kliniken ,automatisch“ jeder Facharztabsolvent die Zusatzbezeichnung „Allergologie“ erwerben kann. Allerdings muß die Zusatzbezeichnung dann auch etwas bedeuten!

In der Versorgung geht der Kampf weiter. Vielen praktizierenden Kollegen steht das Wasser buchstäblich bis zum Hals. Es bleibt abzuwarten, ob die neue Regierung hier andere Akzente setzen will. Eine einfache „Deckelung“ des Budgets kann keine Lösung sein. Seit dem Mittelalter weiß man, daß ein Preisstop für Brot immer zum Verschwinden von Backwaren aus den Bäckereien geführt hat. Trotz der Beteuerungen der Krankenkassen werden die Leidtragenden immer die Patienten sein, die wir mit unserem besten Wissen und Gewissen optimal versorgen wollen.

Auch wenn in Deutschland vielfach geleugnet - immer mehr Entscheidungen fallen bereits heute in Brüssel. In der UEMS (Union Europeen des Medicins specialistes, deutsche Vertreter: B. Hornung, J. Ring) wurde ein Curriculum für einen ,Europäischen Fach-

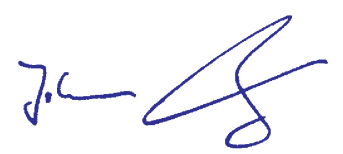

Prof. Dr. Dr. J. Ring arzt für Allergologie“ verabschiedet, das auch den in Mitteleuropa historisch gewachsenen Strukturen der organbezogenen Zusatzweiterbildung Rechnung trägt. Dabei war die Terminologie wichtig. Der deutsche Begriff „Zusatzbezeichnung“ darf nicht einfach als „subspecialty“ übersetzt werden. Es handelt sich vielmehr um eine „additional“ oder ,epi“-specialty!

Eine Ergänzung des „European Allergy White Book" wurde im November 1998 in Brüssel unter der Organisation des „UCB-Institute of Allergy“ (durch den unermüdlichen Einsatz von A. de Weck und U. Wahn) diskutiert. Sie wird im nächsten Jahr den Gesundheits- und Umweltministern in London vorgestellt. Diese Bemühungen haben nicht zuletzt dazu geführt, $\mathrm{da} ß$ Allergie-Forschung im fünften Forschungsrahmenprogramm der EU, welches im neuen Jahr starten soll, namentlich erwähnt wird. In Deutschland hat $\mathrm{H}$. Behrendt als Mitglied des Rates der Sachverständigen für Umweltfragen (SRU) das Allergie-Thema in das im kommenden Jahr zu erstellende neue Umwelt-Gutachten zur Beratung der Bundesregierung gebracht.

Alles im Allem wie in jedem Jahr: Sorgen und Hoffnungen bunt gemischt. ALLERGO JOURNAL hat das ,verflixte siebente Jahr" gut überstanden. Wir wünschen Ihnen frohe und besinnliche Feiertage und viel Schwung und Kraft für die Arbeit im neuen Jahr 1999!

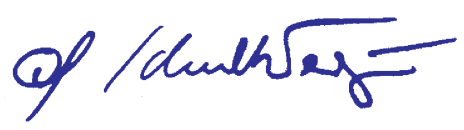

Prof. Dr. G. Schultze-Werninghaus 